\title{
Physics of vacuum at ITEP and around
}

\author{
L.B.Okun \\ ITEP, Moscow, 117218, Russia \\ Email: okun@heron.itep.ru
}

\begin{abstract}
Recollections about a few episodes from the history of physics of vacuum, connected with the names of Pomeranchuk, Landau, Zeldovich, Sakharov and Kirzhnits. The text of the talk will be published in the Proceedings of the International Conference "From the Smallest to the Largest Distances", Tribute to Jean Tran-Thanh-Van, May 24-26, 2001 ("Surveys in High Energy Physics", Taylor and Francis, 2002 , v.16, No.3).
\end{abstract}

The people about whom I will speak - Pomeranchuk, Landau, Zeldovich, Sakharov, Kirzhnits - all of them have passed away. I will also speak about their disciples. I will stress the importance of international exchange of ideas, the crucial role of experiments on $\mathrm{P}$ and $\mathrm{CP}$ violation, the interconnection of particle physics and cosmology.

These three themes are central for my friend Jean Tran-Thanh-Van whose outstanding contribution to physics we are celebrating. They are also central for ITEP with its unique atmosphere. The walls around us have seen the people about whom I will speak and heard their voices.

Lack of time does not allow me to touch upon other important subjects connected with vacuum. They are partly reflected in the text of my talk "Spacetime and Vacuum as seen from Moscow" which has about 90 references (see "2001: A Spacetime Odyssey", Proceedings of the Inaugural Conference of the Michigan Center for Theoretical Physics, University of Michigan, Ann Arbor, 21-25 May 2001, M.J. Duff and J.T. Liu eds., World Scientific, Singapore, 2002; hep-ph/0112031). 
When I became a student of Pomeranchuk in 1950 I heard from him a kind of a joke that the Book of Physics has two volumes: v.1. is "Pumps and Manometers", v. 2. "Quantum Field Theory". Also I heard a statement that vacuum is filled with most profound physical content, which for me was absolutely unclear at that time and a little bit more clear now. In the middle of the 1950's Pomeranchuk formulated his famous theorem about the equality of cross-sections of particles and antiparticles on a given target. In the framework of Regge theory this equality is determined by exchange of a Regge pole with vacuum quantum numbers. This pole was called later on pomeranchukon or pomeron.

In 1956 Pomeranchuk encouraged a group of young people at ITEP to study the paper of T.D. Lee and C.N. Yang on parity non-conservation and to think about it. As a result we (Boris Ioffe, Alexei Rudik and myself) came to the conclusion that the effects predicted by Lee and Yang would mean not only violation of parity, but also violation of charge conjugation invariance between particles and antiparticles. Somewhat later the same result was obtained by Lee, Oehme and Yang.

\section{$* * *$}

We discussed our paper with Lev Landau who usually visited ITEP on Wednesdays, while we visited his Institute of Physical Problems on Thursdays. This was a tradition. Landau was extremely skeptical about the possibility of parity violation. That was during the whole year 1956. The reason for him to be skeptical was that he thought that if parity is violated that would have an imprint on vacuum, because whilst vacuum influences particles, the particles influence vacuum. Hence there should be some asymmetry in vacuum, and the main idea of Landau was that vacuum should be completely symmetric. Therefore he resisted all these attempts, though in 1950 he wrote a paper with Ginzburg on superconductivity in which the notion of spontaneous symmetry breaking was brought into condensed matter physics and a phenomenon, which was later discovered in field theory and is called now Higgs mechanism, was suggested. So, for him it would be quite natural to assume that there is a spontaneous symmetry breaking in vacuum. But 
that's the life, it is not always logical. Thus the fact is that Landau resisted a vacuum which is not symmetric.

Suddenly, just overnight, he came with an idea that parity could be violated, but it should be violated in such a way that particles and antiparticles contribute with opposite sign to the vacuum. And for that there should exist a CP-symmetry: $\mathrm{C}$ for charge conjugation and $\mathrm{P}$ for parity mirror reflection. In that way vacuum would be still symmetric and the whole sin of parity violation will be with particles, not with vacuum. There will exist left-handed neutrino and right-handed antineutrino, but there would be no left-handed antineutrinos or right-handed neutrinos. That was his idea.

The idea that CP is an absolute symmetry was widely accepted, it was beautiful. Personally I was extremely impressed by this solution. However I couldn't understand what's wrong if I write a Lagrangian and put in some places of the Lagrangian some coefficients which are not real but complex, thus introducing corresponding to CP-violation. Therefore I insisted that experiments should be done looking for $\mathrm{CP}$-violating processes. One of them was proposed by Okubo with sigma and antisigma hyperons.

Another one was $K_{L} \rightarrow \pi^{+} \pi^{-}$an obvious candidate. The search for this decay was undertaken in Dubna in the early 1960s in a bubble chamber. They had about 600 3-body decays of $K_{L}^{0}$ and they looked for 2-body decays which was quite easy to discriminate because of missing momentum in the 3 -body decays. It's either neutral pion in $\pi^{+} \pi^{-} \pi^{0}$ or neutrino in semileptonic decays. And there would be just 2 charged pions going back to back in the 2-pion decay. They collected 600 decays and there was not a single 2-body decay. Then the Director of the Lab. stopped this experiment as having no prospect, just waste of time.

If you look into the Particle data booklet, you will find that each 350 3-body decays of $K_{L}$ are accompanied by one 2-body decay of $K_{L}$. Thus by collecting 600 events if they were lucky or if the Director were more patient, they would discover CP-violation. But they were unlucky: the discovery came only two years later and it was done by Fitch, Cronin, Turlay and Christensen.

Thus, the absolute CP-conservation was disproved. 
However the idea of Landau that vacuum must be symmetric pushed Pomeranchuk, Kobzarev and myself to consider a possibility according to which to each of our particles corresponds another particle with exactly the same properties - same mass, spin, charge - everything. And the difference would be only in the phase of CP-violation for these particles. So, what we postulated is the existence of two terms of Lagrangian. One term is the known particles, the other term is these mirror particles, mirror in the $\mathrm{CP}$ sense.

As all interactions between the mirror particles are exactly the same as between our particles, there should be mirror atoms, mirror molecules, mirror condensed matter, mirror stars and even mirror life. And all this would be unobservable, because there was no interaction of the electromagnetic, weak, or strong type which would connect these two worlds. The only interaction which connected them (we were forced to this conclusion) was gravitational one. Because if there were separate gravitons in the two worlds, then it would be not a scientific assumption. It's just a matter of faith, but not of science. It couldn't be scientifically proved or disproved.

There were many papers and they still appear now. But I am not especially enthusiastic about it for many years, because it seems to me there are other better ideas: namely, to accept the asymmetry of vacuum and not to insist on the symmetry of it.

My next subject is connected with cosmological term. All of you know that cosmological term was introduced by Einstein in 1916 and that later he considered it as a blunder and there were a lot of discussions whether it should be or should not be. You certainly heard about them.

It is paradoxical, but it took many years before the first paper appeared in which it was proven that it should be (not in a mathematical way, but in physical, by Yakov Zeldovich). If you have quantum mechanics, then there must be in the framework of general relativity a cosmological term due to virtual particles. In other words, due to zero state quantum oscillations.

Zeldovich was the first who published estimates of the cosmological term. His first estimate was that it must be more than 120 orders of magnitude larger than the upper limit on it. Then he tried to invent various expressions 
which would contain not $m_{P l}^{4}$, but somehow to get $m_{P l}$ in denominator to make it proportional to the gravitational coupling constant.

Moreover Zeldovich was the first to notice and to publish in 1968 the observation that the contribution of bosons and fermions to the cosmological term are of opposite sign, and therefore in principle if there is boson-fermion symmetry, they would cancel each other. If the symmetry is broken then they would cancel partly.

This was a few years before the time when Yuri Golfand and Evgeny Likhtman suggested that there exists supersymmetry. In 1968 nobody spoke about symmetry between fermions and bosons.

Here I would like to make a digression and to tell you about another piece of work done in an office which is adjacent to my office at ITEP.

There in 1965 Mikhail Terentyev discovered together with Vladimir Vanyashin (Vanyashin is a physicist from Ukrain who still comes from time to time to ITEP, while Terentyev died a few years ago at the age of 60) that, unlike in quantum electrodynamics, in nonabelian theories vacuum polarization has a negative sign. This result has been derived again by Iosif Khriplovich in 1969. There is so called ghost in the gauge theories. If you draw Feynman diagrams, you cannot limit yourself with propagators of usual particles only. You have to add propagators of ghosts which were introduced by Fadeev and Popov in 1966. In 1965 they were not known to Vanyashin and Terentyev. Therefore their result was not 22 in nominator, but 21.

This mistake was corrected by Khriplovich, who again not using the ghost but using the so-called Coulomb gauge derived the correct result. But neither they, nor the people around them realized what a great discovery was made by these people. Personally I didn't understand and my colleagues didn't understand, and nobody understood at that time. The discovery became clear after Gell-Mann introduced the notion of color, after QCD was formulated and after Gross, Wilczek and Politzer coined the term "asymptotic freedom": this negative sign in nonabelian gauge theories leads to decrease of $\alpha$, of the square of charge, to zero at large momentum transfers. I have to admit that we were not attentive enough to what was going on at that time in 1969 at SLAC, to deep inelastic scattering, to partons. 
Another important contribution which was made at ITEP is the so-called QCD sum rules, sometimes called ITEP sum rules. They were written by Mikhail Shifman, Arkady Vainshtein and Valentin Zakharov in the late 1970s. They exploited the fact that the gauge coupling constant $\alpha$ not only decreases at short distances, but also increases at large distances. And as a result at large distances (large means $10^{-13} \mathrm{~cm}$ ) the strong interaction becomes really strong and nonperturbative. One cannot apply perturbation theory to it. They managed to write QCD sum rules in which vacuum expectation values of gluonic fields and quark fields were introduced. There are now, I believe, a few thousand papers which develop this theoretical discovery.

Sakharov didn't work at ITEP, he was at Lebedev Institute. And from Lebedev Institute he went to a secret city Arzamas-16 (now it is called Sarov) where he became "the father of the Russian hydrogen bomb". Then he returned back to Moscow in the 60s, just after the discovery of CP-violation. He was practically uneducated in particle physics, and it was astonishing to see how fast he went to the heart of the subject.

I already mentioned that the decays of $\Sigma$ hyperons and $\bar{\Sigma}$ hyperons are different when CP is violated. Sakharov used this difference as a prototype. What is the characteristic for these decays? That strangeness is violated: $\Sigma$ hyperons are strange while the products of their decay (protons and pions) are nonstrange. Sakharov went from weak interactions with strangeness violation directly to the idea of baryon number violation and from $\Sigma$ decays to the Universe. He used a similar mechanism for the early Universe in order to explain why we are built from particles and not antiparticles. Or, better to say, why there is not an equal amount of particles and antiparticles in our world. In the latter case we would not exist at all, because particles and antiparticles would annihilate and there would be nothing to build our matter from. Protons and antiprotons, electrons and positrons would annihilate, and there will be a world full of neutrinos and photons.

Thus, he started with hot symmetric Universe, big bang Universe. And he explained how could it be that from this symmetric Universe in which 
the number of particles and antiparticles were equal, our world appeared. His idea was that this occurred due to CP-violation, to baryon number nonconservation and to non-equilibrium state in which this primordial soup was cooling due to the expansion of the Universe. Because of these three reasons there appeared a tiny excess of baryons compared to antibaryons. This tiny excess survived because there were baryons to which there was no match to annihilate with. While the majority ((majority means a billion of baryons and antibaryons) would annihilate, one baryon would stay alone and survive. And that's how he explained our existence.

I believe, this paper, only a few pages, is one of the most daring papers of the last century.

The idea of baryon number nonconservation was necessary for Sakharov, but it was not provided by the theory which existed at that time. Later on a number of mechanisms were theoretically discovered which lead to baryon number nonconservation. At present there are several mechanisms, how to violate baryon number conservation. But unfortunately they create new problems because through them the asymmetry which could be produced at the early stages of the Universe could be destroyed at the late stages.

The name Sakharov is quite famous and the name Kirzhnits is not so famous. David Kirzhnits was a colleague of Sakharov also at Lebedev Institute. I remember how in 1972 he told me (we were at the Conference in Tashkent) about his idea of phase transitions in vacuum. That was absolutely new to me. I could understand face transition in some material medium, but not in vacuum. At that time the gauge symmetry $S U(3) \times S U(2) \times U(1)$ broken by Higgs mechanism was already known. And the idea of Kirzhnits was that if we go backward in time, then at the stage of hot Universe, at very high temperatures, the symmetry would be restored and all massive particles would become massless. There would be no Higgs vacuum expectation value in vacuum. This idea was further developed later on by Kirzhnits and his student Andrei Linde.

A few years later Igor Kobzarev, Yakov Zeldovich and myself used this idea of phase transitions when considering a model of CP-violation proposed by T.D. Lee. In this model CP is spontaneously broken. The Lagrangian is CP-invariant. However, its potential has two minima: at one of them a 
neutral pseudoscalar field $\phi$ has a classical solution $\phi=+\phi_{0}$ while another one $-\phi_{0}$. This expectation values lead to CP-violation with the sign of CPviolation being different in these two minima. Thus, if the Universe chooses the first minimum, when it cools down, the phase would be plus in all CPviolating effects; if it chooses the second minimum, the phase would be minus. But various parts of the Universe at early stages are not casually connected, they cannot "talk" to each other. Therefore, each point has to choose its sign for himself (or itself, herself - I don't know). As a result you get a kind of a chessboard: you have regions with plus sign and regions with minus sign. More precisely it looks like a continent in which there are sees and there are islands in the sees and there are lakes on the islands and islands in the lakes and so on. All these different vacuum domains are separated by walls. It's very easy to understand: if you have in one point the first vacuum and in another point the second one, you will get large potential energy between them. If the border region is thick enough, you will have a lot of energy in the border. If you try to squeeze this border, you will find that you cannot squeeze it too much due to uncertainty principle. You end up with an absolutely new kind of a solution in field theory.

We are used to consider field theory in terms of particles, microscopic objects. These borders, these walls, how we called them, were even not macroscopic objects, they were megascopic objects, they were huge. We considered the cosmology of Universe filled with such walls and the cosmology turned out to be very unusual: the expansion of the Universe was much faster than in the Friedmann model: the wall which is the closest to us had to go far behind the horizon. But it could manifest itself by perturbing the black body radiation. We don't seat in the center of our domain, there is no reason for us to be at the center, we are ordinary people. Therefore we are closer to one of the walls and then there must be an asymmetry of black body radiation. But there is no such symmetry in Nature.

The domain wall was the first megascopic solution in quantum field theory. Soon afterwards other solutions appeared. A.Polyakov and G.'t Hooft discovered monopoles in $\mathrm{SU}(2)$, T.W.B.Kibble discovered strings, not microscopic strings, not superstrings, but cosmological strings which go through the whole Universe. And there is a whole theoretical industry of such solutions.

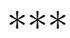


In 1974 T.D.Lee and G.C.Wick considered another model in which the potential of the field $\phi$ was slightly asymmetric. Therefore one of the two minima was lower and stable, while the other one was higher and metastable. If the Universe were in higher place, it could tunnel to lower place.

What does it mean: the Universe can tunnel? Assume that at a given place you have the upper vacuum; then you will gain energy, if you go at that place to the lower vacuum. Thus, at first sight a simple question arises: Why not to go to a lower vacuum here and now? The answer is that between two vacua there is a wall which I've already described before: a material wall, very heavy, very dense. You need energy to create it. Therefore there exists a critical radius $R_{c}$ at which the gain in energy in volume of a bubble is becoming larger than the loss of energy used for creation of the surface of the bubble.

It was a very frightening experience which I had when I first thought about these bubbles. I thought about the possibility that at some collider the collision of two particles would enhance the probability of creating such a microbubble. And if a bubble of critical dimension is produced, then it can expand infinitely, because the volume energy goes like $r^{3}$ and the surface energy - like $r^{2}$. Thus, for large $r$ volume will predominate. And very soon the wall of the bubble would move with a velocity of light, and the bubble would expand and destroy the whole world. I really shivered when I thought about this. But then I somehow relaxed by thinking about the past: that Universe was hot, there was a lot of various collisions in it. Therefore if bubbles could be produced by collisions, then they already were produced and we are living in a true vacuum now.

A few months later I told Andrei Sakharov about these bubbles. I vividly remember his reaction. He said: "Such theoretical work should be forbidden. It's too dangerous". I tried to persuade him with arguments about past of the Universe and all that. And he said: "Nobody had collided two lead nuclei in the Universe". I took this quite seriously.

But in 1984 a paper by P. Hut appeared, who considered the same problem. Our conversation was in 1974 and 10 years later this paper appeared. Hut had estimated a number of uranium-uranium collisions in the Universe during all these billions of years of its existence. Then other people estimated. They found (this was done when RHIC at Brookhaven started to be discussed) that during the existence of Universe there were enough lead-lead, iron-iron, uranium-uranium and even gold-gold collisions. There were about $10^{47}$ iron-iron collisions, while RHIC would produce at most $10^{12}$ through its 
lifetime. Thus, we are 30 orders of magnitude safe.

I would like to conclude by saying that there was an enormous progress during last 50 years in the understanding of physics in general and physics of vacuum in particular. As a result of this progress important problems have been solved, but the number of unsolved problems facing us has increased immensely. I hope that there will be even greater progress in the next 50 years.

Thank you. 